\title{
Pandemic affects the uninfected: hypercalcemia in a peritoneal dialysis patient due to protective equipment shortage
}

\author{
Sanjeev Shrestha ${ }^{1} \cdot$ Raza Zaidi $^{1} \cdot$ Conrad Schuerch $^{2} \cdot$ Gurmukteshwar Singh ${ }^{3} \mathbb{C}$
}

Received: 26 December 2021 / Accepted: 1 February 2022 / Published online: 25 February 2022

(c) The Author(s) under exclusive licence to Italian Society of Nephrology 2022

Keywords Hypercalcemia · Peritoneal dialysis · COVID-19 · Effects on uninfected · PPE shortage · Drywall dust · Granulomatous lung disease

\section{The Case}

A 59-year-old male with end-stage kidney disease (ESKD) from diabetes, human immunodeficiency virus (HIV) infection, coronary artery disease, and hypertension was stable on continuous cycler peritoneal dialysis for about 2 years. His mineral bone disorder had been well controlled on calcium acetate $667 \mathrm{mg}$ with meals, $0.25 \mathrm{mcg}$ calcitriol and 1000 Units of cholecalciferol daily. In the setting of worsening hyperphosphatemia, attributed to dietary indiscretion at the time, calcium acetate was increased and calcitriol stopped. His health insurance did not cover non-calciumbased phosphate binders.

Four months later, he developed intermittent subtle confusion, impaired balance and stuttering. Corrected calcium level was found to be $12.9 \mathrm{mg} / \mathrm{dL}$, phosphorus $6.9 \mathrm{mg} / \mathrm{dL}$ and PTH $9 \mathrm{pg} / \mathrm{mL}$. Other significant lab values included 25 -hydroxy vitamin D level $28 \mathrm{ng} / \mathrm{mL}, 1,25$-dihydroxy vitamin D level $39 \mathrm{pg} / \mathrm{mL}$ (reference range $18-72 \mathrm{pg} / \mathrm{mL}$ ), PTH-related peptide (PTHrP) $62 \mathrm{pg} / \mathrm{mL}$ (reference range $14-27 \mathrm{pg} / \mathrm{mL}$ ) and angiotensin converting enzyme (ACE) was elevated at 111 Units/L (reference range 9-67 Units/L). Thyroid stimulating hormone was normal. His hypercalcemia presentation and clinical course are summarized in Fig. 1.

Gurmukteshwar Singh

gsingh3@geisinger.edu

1 Department of Medicine, Geisinger Health, Danville, PA, USA

2 Department of Pathology, Geisinger Health, Danville, PA, USA

3 Department of Nephrology, Geisinger Health, 100 N. Academy Blvd, Danville, PA 17822, USA
Calcium acetate was discontinued. CT chest showed numerous conglomerations of centrilobular nodules in multiple lobes of both lungs (Fig. 2A). Besides mild intermittent cough, he denied any related symptoms. He underwent bronchoscopy with transbronchial biopsy. Surgical pathology showed several granulomas with small aggregates of giant cells and associated lymphocytes (Fig. 2C). There were calcified inclusions in some of the giant cells and polarizable crystalline material within most of the giant cell granulomas (Fig. 2D).

Pneumocystis jirovecii smear, bacterial, mycobacterial and fungal cultures of the bronchoalveolar lavage were negative. Nucleic acid amplification tests for Mycobacterium avium complex, respiratory viruses, and severe acute respiratory syndrome coronavirus 2 (SARS-CoV-2) were negative. HIV continued to be well-controlled. Myeloperoxidase and proteinase-3 anti-neutrophil cytoplasmic antibodies were negative.

During nephrologist-led interviews, he complained about the N95 mask shortage during the pandemic. He had been renovating his home over 6 months, sanding drywall without any respiratory protective equipment. Within a few weeks of stopping this, his calcium level normalized. He has subsequently been restarted on calcium acetate capsules to manage hyperphosphatemia. Follow-up CT chest after 4 months showed complete resolution of lung nodules (Fig. 2B). A year later, the patient is asymptomatic and on peritoneal dialysis. ACE levels have normalized at 53 Units/L. 1,25-Dihydroxy vitamin levels went down to $8 \mathrm{pg} / \mathrm{mL}$. His most recent PTH was $358 \mathrm{pg} / \mathrm{mL}$, with corrected calcium $8.8 \mathrm{mg} / \mathrm{dL}$ and phosphorus $4.9 \mathrm{mg} / \mathrm{dL}$. 


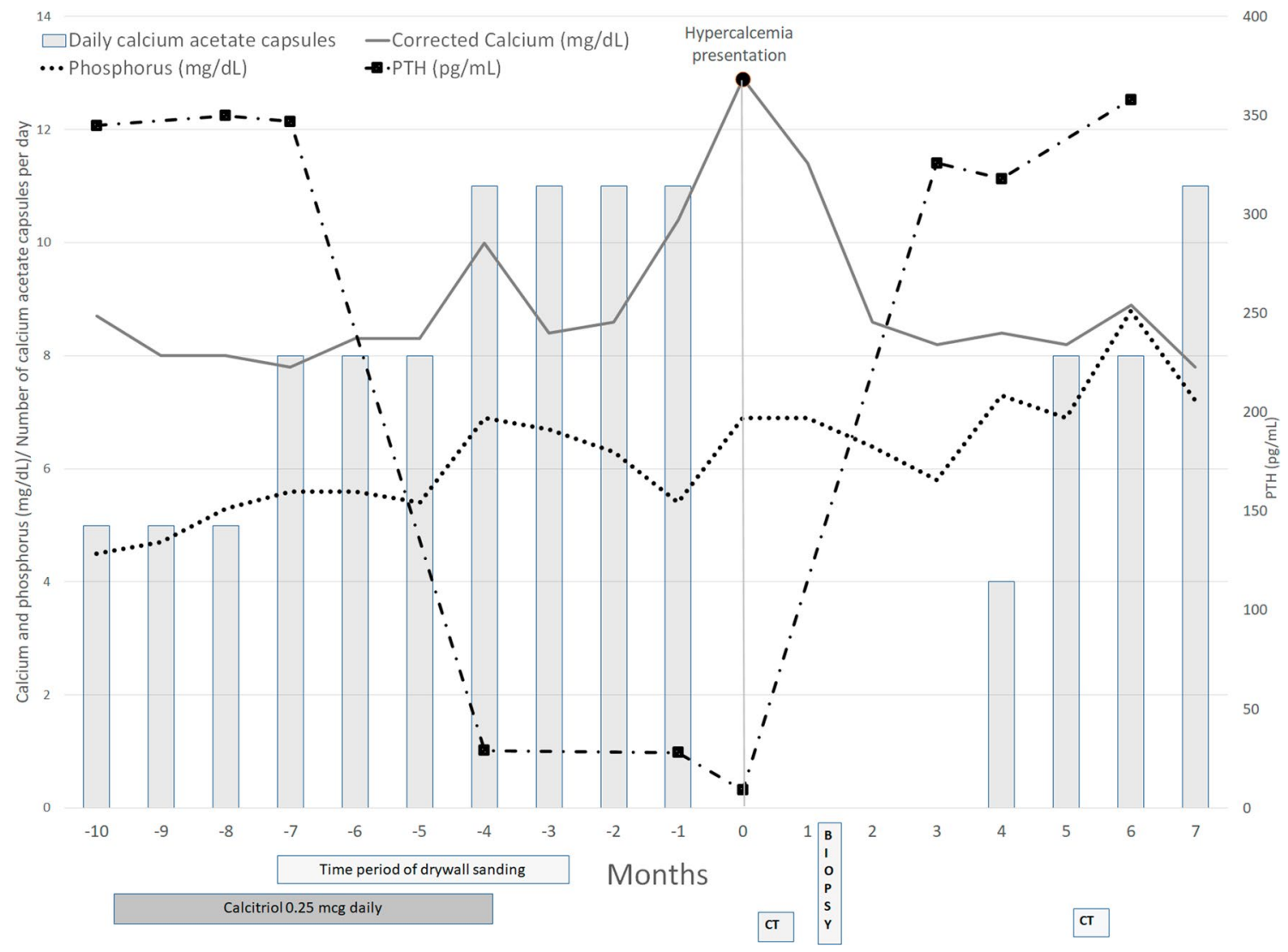

Fig. 1 Summary of case, clinical interventions and laboratory value progression: the solid line, dotted line and interrupted line with square markers indicate the patient's serum calcium, phosphorus and parathyroid hormone, respectively, throughout his clinical course. Time (in months) is depicted along the $\mathrm{x}$-axis. The index presentation with hypercalcemia occurred at month 0 . The $y$-axis represents serum

\section{Lessons for the clinical nephrologist}

\section{How to determine the etiology of hypercalcemia in a dialysis patient?}

Hypercalcemia has been reported in $5.1 \%$ of patients undergoing maintenance peritoneal dialysis. It is associated with significantly increased mortality and morbidity [1]. The most common cause is tertiary hyperparathyroidism due to mineral bone disorder [2]. Other common causes include use of calcium-based phosphate binders or vitamin $\mathrm{D}$ analogues, malignancy, and immobilization. Unfortunately, few comprehensive hypercalcemia evaluation algorithms specifically address dialysis patients [3]. This case illustrates the use of our diagnostic algorithm specific to dialysis patients, as summarized in Fig. 3. Although our patient had amodestly elevated PTHrP, the elevation of C-terminal PTHrP has been well described in patients with impaired kidney function and hypercalcemia without cancer [4]. Due to negative cancer calcium and phosphorus (in $\mathrm{mg} / \mathrm{dL}$ ). The number of calcium acetate capsules the patient was taking per day is also represented along the $y$-axis and by vertical bars in the figure. The secondary y-axis represents the patient's PTH (in $\mathrm{pg} / \mathrm{mL}$ ). Various clinical events, like the two CT scans, transbronchial biopsy, as well as time periods for drywall sanding and calcitriol use are indicated below the $\mathrm{x}$-axis

screening, an established alternative pathological cause, and symptom self-resolution, we did not pursue N-terminal PTHrP testing. Working through our algorithm systematically, normal 1,25-dihydroxyvitamin D level (despite ESKD), imaging and histology established the diagnosis of granulomatous disease.

\section{Which granulomatous disease can causehypercalcemia and how do I work through the differential diagnosis?}

In granulomatous disease, hypercalcemia occurs due to increased vitamin D activation from 1-alpha-hydroxylase [5]. While 1,25-dihydroxyvitamin $\mathrm{D}$ level is expected to be low in ESKD, in our patient, it was normal [6]. The true extent of 1-alpha-hydroxylase activity became apparent in hindsight: his 1,25-dihydroxyvitamin D level was only $8 \mathrm{pg} /$ $\mathrm{mL}$ once the granulomas resolved. Hypercalcemia has been described in the setting of sarcoidosis, mycobacterial or fungal infection, granulomatosis with polyangiitis, Crohn's 


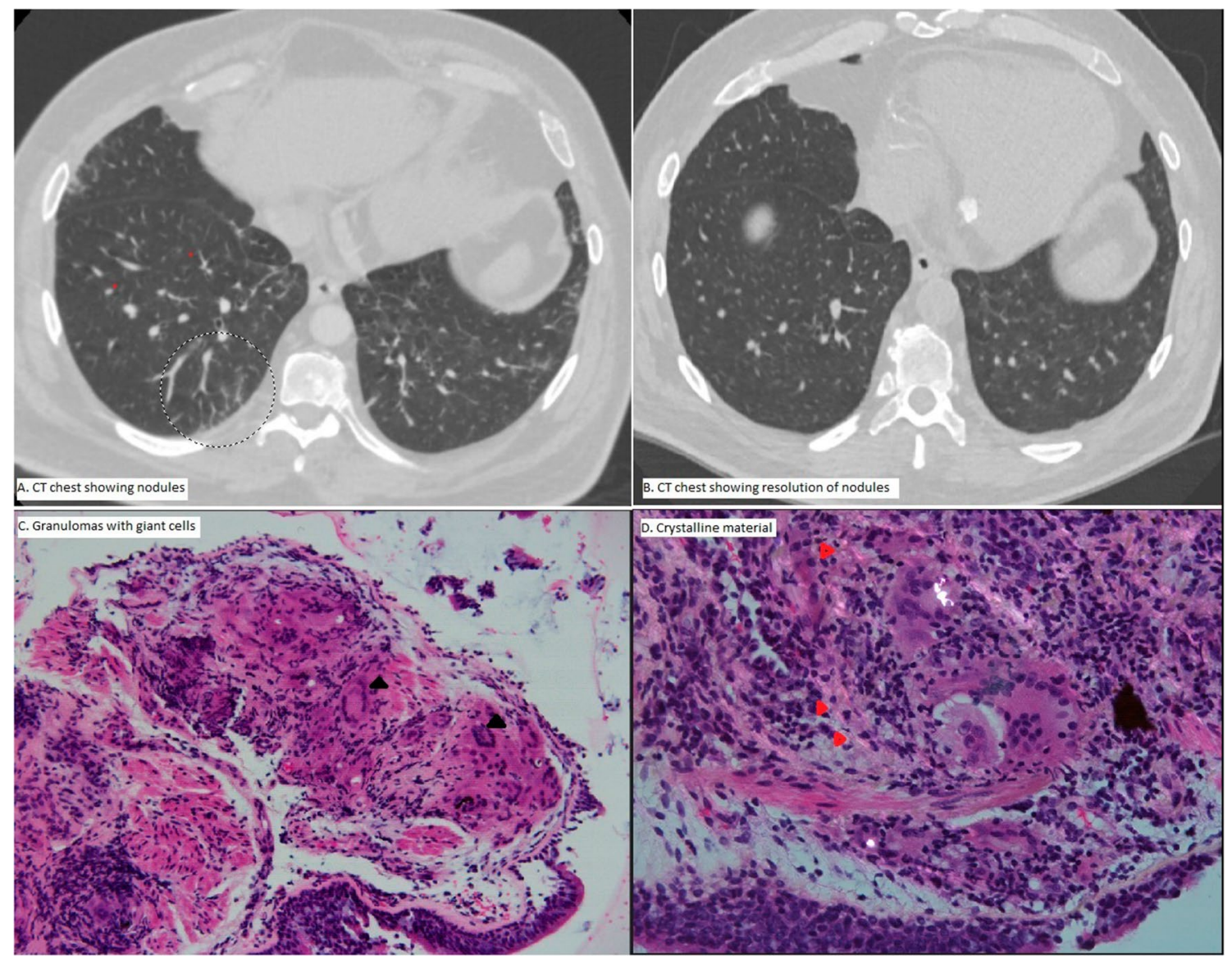

Fig. 2 A Initial chest CT with bilateral granulomatous changes in the lungs. B Chest CT performed 4 months later with resolution of these changes at the same lung level. $\mathbf{C}$ A hematoxylin and eosin stain of a section of the transbronchial biopsy showing giant cell granulo-

disease, hepatic granulomatosis and granulomatosis associated with exposure to talc and toxic substances [5]. After making a diagnosis of hypercalcemia due to granulomatous lung disease, determination of etiology is critical. In a comparative study of 142 patients with hypersensitivity pneumonitis, the inability to identify an inciting agent was an independent predictor of shorter survival [7].

Nephrologists may not feel comfortable determining the etiology of granulomatous disease in hypercalcemic patients. This work-up is better described in pulmonology literature and frequently requires multidisciplinary evaluation [8]. Ruling out infectious etiologies was the first step as our patient also had HIV infection. Granulomatous vasculitis serology was also negative. In clinical work-up, elevated ACE level is frequently stated to support the possibility of sarcoidosis. However, in a population-based study, elevated ACE levels had a positive predictive value of only $25.4 \%$ and a false positive rate of $10 \%$ for sarcoidosis [9]. Our patient did not have a high sarcoidosis diagnostic score, which is more reliable for this diagnosis [S1]. As prior immunofluorescence mas. Multinucleated giant cells are indicated by black triangles. D A section of the transbronchial biopsy under polarized light, showing polarizable crystalline material in the granulomas, indicated by red arrows (colour figure online)

localization studies described, the elevated ACE levels in our patient were likely a non-specific marker of epithelioid and giant cell-rich granulomas, and improved with their resolution [S2].

After ruling out these etiologies, pneumonitis from environmental exposure remains the leading differential diagnosis. The triggering factor can be extremely difficult to pinpoint but identification is critical [S3]. The calcium-containing inclusions and crystalline material in non-necrotizing pulmonary granulomas, though striking in appearance, are non-specific. A case series of six patients described sarcoidosis in half of the cases. Atypical mycobacterial infection was determined to be the cause in one of the cases [S4]. While immunoglobulin crystal storage has been reported in histiocytosis in 13 patients, it was associated with a lymphoplasmacytic neoplasm, had multi-system involvement, and would not be not selfresolving [S5]. In this absence of histopathologic specificity, history-taking becomes exceptionally important in identifying the underlying etiology. 


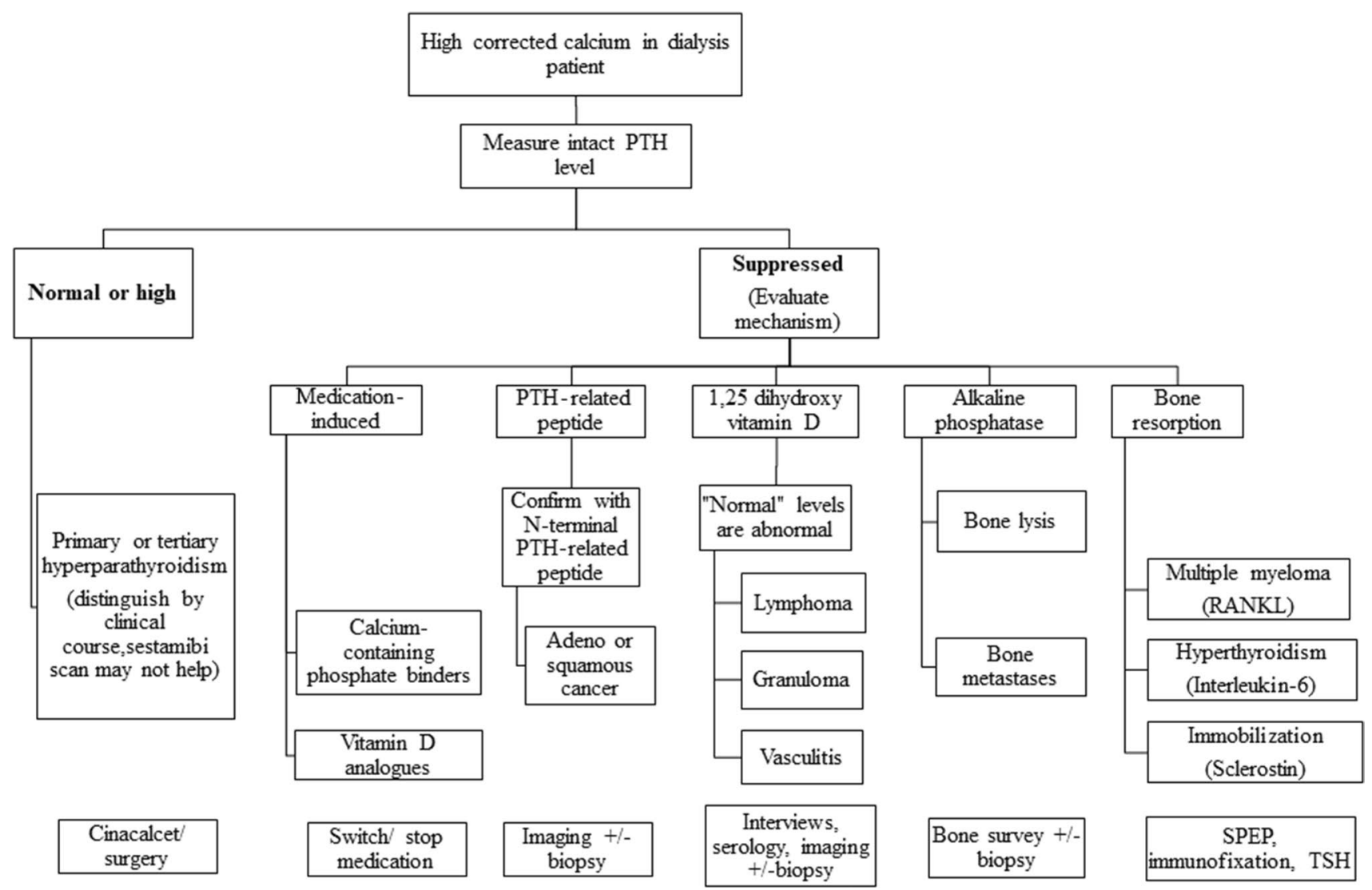

Fig. 3 Determining etiology of hypercalcemia in dialysis patients producing little urine: work-up algorithm for ascertaining etiology of hypercalcemia by following a mechanism-based work-up. The lowest

It is critical that nephrologists become more familiar with standardized interviews to systematically detect environmental exposure [S3]. Dialysis patients have long-term established relationships and more frequent contact with nephrologists. Hence, nephrologists are better positioned to establish trust and to obtain a more complete history. Of note, our patient did not reveal his environmental exposure during pulmonology interviews. His nephrologist was able to obtain this esoteric history during a structured interview, which led to identification of drywall dust as the culprit [S3]. Drywall joint compounds are made from many ingredients known to trigger granulomatous inflammation like talc, calcite, mica, gypsum and silica [S6]. Once the exposure history was established, we were able to counsel him appropriately, eventually leading to resolution of hypercalcemia.

boxes in the figure indicate the next steps to confirm/manage the etiology. RANKL receptor activator of nuclear factor- $\mathrm{\kappa B}$ ligand, SPEP serum protein electrophoresis, $T S H$ thyroid stimulating hormone

\section{How has the SARS-CoV-2 pandemic affected our non-infected patients?}

Severe acute respiratory syndrome coronavirus 2 has been responsible for an ongoing global pandemic of coronavirus disease 2019 (COVID-19), resulting in significant morbidity and mortality among dialysis patients. In a national analysis, infected dialysis patients had a mortality rate of $24.9 \%$ [S7]. The indirect effects on non-infected patients are less well recognized. Nearly 1 in 3 excess deaths in the United States during the pandemic were not attributed to COVID-19. While some excess deaths could be misattributed, the large observed increase in death from non-infectious conditions indicates an under-recognized indirect effect [S8]. Secondary pandemic mortality could be a result of delayed access to healthcare and worsening social determinants of health. The early period of the pandemic was marked by severe shortages of medical supplies in the United States, the world's largest importer of personal protective equipment [S9]. Our report shows that downstream effects like protective equipment shortages may have a significant health impact on frail 
dialysis patients. Further studies evaluating these indirect effects on susceptible populations are sorely needed.

In conclusion, a nephrologist-led systematic approach to evaluation of hypercalcemia in our dialysis patient resulted in the extremely rare diagnosis of granulomatosis from drywall dust inhalation. We also identify a need to further study the indirect effects of the COVID-19 pandemic on non-infected patients.

Supplementary Information The online version contains supplementary material available at https://doi.org/10.1007/s40620-022-01272-6.

\section{Declarations}

Conflict of interest The authors have no conflicts of interest in relation to this manuscript. No funding was received.

Ethical approval Our patient provided written consent for publication of this case report published in the hope that others may learn and benefit from his story.

\section{References}

1. Rivara MB, Ravel V, Kalantar-Zadeh K et al (2015) Uncorrected and albumin-corrected calcium, phosphorus, and mortality in patients undergoing maintenance dialysis. J Am Soc Nephrol. https://doi.org/10.1681/ASN.2014050472

2. Cunningham J, Locatelli F, Rodriguez M (2011) Secondary hyperparathyroidism: pathogenesis, disease progression, and therapeutic options. Clin J Am Soc Nephrol. https://doi.org/10. 2215/CJN.06040710

3. Mf C, Ds S (2003) A practical approach to hypercalcemia. Am Fam Physician 67(9):1959-1966

4. Lum G (2011) Falsely elevated parathyroid hormone-related protein (PTH-RP) in a patient with hypercalcemia and renal failure. Lab Med. https://doi.org/10.1309/LM23TD4IUTAJBJMG

5. Jacobs TP, Bilezikian JP (2005) Clinical review: rare causes of hypercalcemia. J Clin Endocrinol Metab. https://doi.org/10.1210/ jc. $2005-0675$

6. Dusso AS (2011) Kidney disease and vitamin D levels: 25-hydroxyvitamin D, 1,25-dihydroxyvitamin D, and VDR activation. Kidney Int Suppl. https://doi.org/10.1038/kisup.2011.30

7. Fernández Pérez ER, Swigris JJ, Forssén AV et al (2013) Identifying an inciting antigen is associated with improved survival in patients with chronic hypersensitivity pneumonitis. Chest. https:// doi.org/10.1378/chest.12-2685

8. Ohshimo S, Guzman J, Costabel U, Bonella F (2017) Differential diagnosis of granulomatous lung disease: clues and pitfalls: number 4 in the series "Pathology for the Clinician" Edited by Peter Dorfmüller and Alberto Cavazza. Eur Respir Rev. https://doi.org/ 10.1183/16000617.0012-2017

9. Ungprasert P, Carmona EM, Crowson CS, Matteson EL (2016) Diagnostic utility of angiotensin-converting enzyme in sarcoidosis: a population-based study. Lung. https://doi.org/10.1007/ s00408-015-9826-3

Publisher's Note Springer Nature remains neutral with regard to jurisdictional claims in published maps and institutional affiliations. 